\title{
Displacement field of the Italian area from permanent GPS stations
}

\author{
Federica Riguzzi $\left({ }^{1}\right)$ and Antonio Zanutta $\left({ }^{2}\right)$ \\ ( $\left.{ }^{1}\right)$ Istituto Nazionale di Geofisica, Roma, Italy \\ $\left.{ }^{2}\right)$ DISTART, Università di Bologna, Italy
}

\begin{abstract}
A network of nine permanent GPS stations, six of them located in Italy (Cagliari, Matera, Medicina, Noto, Padova and Torino), the other three in Central Europe (Graz, Zimmervald and Wettzell) was been analyzed four times from October 1996 till 1998. After the GPS data processing, a rigorous statistical analysis based on the $F$ (Fisher) test applied on the detected coordinate differences showed significant displacement at Matera, Medicina and Noto. The mean velocities computed for these sites with respect to Wettzell are $(0.6 \pm 0.3)$ $\mathrm{cm} / \mathrm{yr}$ for Matera, $(0.7 \pm 0.2) \mathrm{cm} / \mathrm{yr}$ for Medicina and $(0.6 \pm 0.3) \mathrm{cm} / \mathrm{yr}$ for Noto. GPS velocities agree with those derived by VLBI and ITRF96 solutions, provided error ellipses are taken into account. The deformation analysis of the last time span shows a suspicious horizontal jump at Padova of $(1.0 \pm 0.1) \mathrm{cm}$ corresponding to an antenna changing within the same period. This fact shows, in spite of the care used in permanent GPS installation, how the results of the deformation analysis may be strongly conditioned by site problems.
\end{abstract}

Key words GPS permanent stations - displacement field - Italian region - statistical analysis

\section{Introduction}

The aim of this paper is to estimate the displacements and velocities of GPS tracking sites located in Italy with respect the some of them located in the interior of stable Europe.

To reach this goal, a network of nine GPS sites was repeatedly analyzed in time. This network is composed of six permanent Italian stations Cagliari: (CAGL), Matera (MATE), Medicina (MEDI), Noto (NOTO), Torino (TORI), Padova (UPAD) and three others located outside the Italian peninsula: Graz (GRAZ), Wettzell (WTZR) and Zimmervald (ZIMM).

Mailing addresses: Dr. Federica Riguzzi, Istituto Nazionale di Geofisica, Via di Vigna Murata 605, 00143 Roma, Italy; e-mail: riguzzi@ing750.ingrm.it - Dr. Antonio Zanutta, DISTART, Università di Bologna, Viale Risorgimento 2, 40136 Bologna, Italy; e-mail: antonio.zanutta@mail.unibo.ing.it
The originality of the present work is that a rigorous statistical analysis to detect the displacement significance is applied to the Italian tracking site network for the first time. TORI (North-Western Italy), started to work in 1996: no displacement trend or velocity are at present available, not being included in the ITRF (IERS Terrestrial Reference Frame) computations and, from a geophysical point of view, it is the Italian permanent GPS station located north of the Apennines, far from the Adriatic area and seismically active regions.

Moreover, it may be useful to verify if the analysis of limited GPS data sets may led equally to meaningful conclusions on the deformation trends of the Italian peninsula with respect to those achieved from prolonged time intervals or by other geodetic techniques.

With this aim, four time spans were selected from 1996 to 1998, choosing five observing days within four GPS weeks 877 (1996), 900 (1997A), 929 (1997B), 945 (1998), as listed in table I. 
Table I. GPS observations.

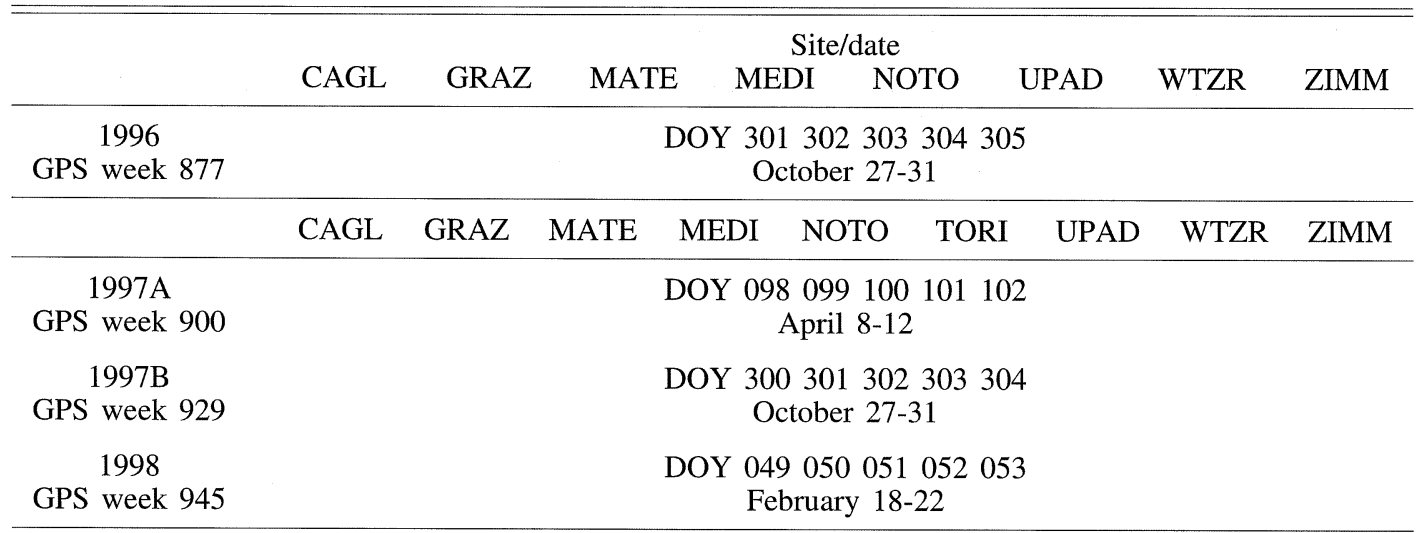

\section{Data analysis}

The main steps of the data analysis consist of the GPS data processing, the network adjustments, the comparison between the network solutions (after the estimation and removal of systematic effects due to scale and rotations) by a statistical analysis to evaluate the significance of the observed coordinate differences and finally the computation of the $2 \mathrm{D}$ vector displacements and the corresponding error ellipses.

GPS data processing was performed by the Bernese GPS software version 4.0 developed at Bern University (Rothacher et al., 1996) following the user manual suggestions. Precise ephemeris, satellite clock corrections, antenna height phase center variations and some other general files provided by the CODE center at Bern University were included in the computation. Tropospheric delay parameters every four hours were estimated for each site starting from the Saastamoinen standard model.

Each observing DOY (Day Of Year) was processed separately obtaining one network solution (coordinates, tropospheric delays and covariance matrix) per DOY. At the end of the GPS data processing we have five network solutions per GPS week (total of 20 networks).

The five network solutions of each GPS week were minimal constrained adjusted by NETGPS, a software suitably implemented to analyze GPS networks (Crespi, 1996).
Table II shows some global parameters of each network adjustment, the equation number, the number of estimated unknowns, the total redundancy, the variance of unit weight $\sigma_{0}^{2}$ ( $a$ priori and a posteriori) values and the experimental and threshold values of $\chi^{2}$. Table III shows the maximum and mean error and reliability ellipse semiaxes, computed on the local tangent plane and the corresponding mean confidence interval on height at $99 \%$ confidence level. The planar error ellipse semiaxes range within $0.5 \mathrm{~cm}$, whilst the confidence interval on height determinations results below $1.9 \mathrm{~cm}$. The site reliability depends substantially on the network geometry, precision and local observation redundancy; it represents the maximum error on the coordinate estimates due to the maximum hidden (undiscovered) error. The planar reliability ellipse semiaxes range, at $99 \%$ confidence level, within $1.7 \mathrm{~cm}$, whilst the height, obviously the worst determinable component, has a mean confidence interval on height estimates not greater than $7.8 \mathrm{~cm}$.

To account for possible residual systematic effects on coordinate differences generally due to ephemeris errors (Bányai, 1991; Crespi et al., 1993), four parameters (scale and rotations) between pairs of coordinate sets were estimated with respect to the network baricentrum. Thanks to the very good satellite ephemeris quality provided by CODE, no significant scale and rotation parameters were found in the first two comparisons, whilst small significant com- 
Table II. Global parameters of the network adjustments. $\sigma_{0}^{2}=$ variance of unit weight; Eq. = equations;
Unk. = unknowns; Red $=$ redundancy.

\begin{tabular}{cccccccc}
\hline \hline $\begin{array}{c}\text { GPS } \\
\text { week }\end{array}$ & $\begin{array}{c}\text { A priori } \\
\sigma_{0}^{2}\end{array}$ & $\begin{array}{c}\text { Estimated } \\
\sigma_{0}^{2}\end{array}$ & $\begin{array}{c}\text { Threshold } \\
\chi^{2}(a=1 \%)\end{array}$ & $\begin{array}{c}\text { Experimental } \\
\chi^{2}\end{array}$ & Eq. & Unk. & Red. \\
\hline 877 & 1.00 & 1.01 & 117.06 & 85.90 & 105 & 24 & 84 \\
900 & 1.00 & 1.04 & 131.14 & 104.28 & 120 & 27 & 96 \\
929 & 1.00 & 1.00 & 127.63 & 93.86 & 117 & 27 & 93 \\
945 & 1.00 & 1.00 & 131.14 & 95.87 & 120 & 27 & 96 \\
\hline
\end{tabular}

Table III. Precision and reliability at 99\% confidence level. SMAX, RMAX and SMED, RMED are respectively the maximum, and mean values of the error and reliability ellipse semiaxes; INT $h$ and INT Rh the corresponding confidence and reliability mean intervals on height.

\begin{tabular}{ccccccc}
\hline \hline $\begin{array}{c}\text { GPS } \\
\text { week }\end{array}$ & $\begin{array}{c}\text { SMAX } \\
(\mathrm{cm})\end{array}$ & $\begin{array}{c}\text { SMED } \\
(\mathrm{cm})\end{array}$ & $\begin{array}{c}\text { INT h } \\
(\mathrm{cm})\end{array}$ & $\begin{array}{c}\text { RMAX } \\
(\mathrm{cm})\end{array}$ & $\begin{array}{c}\text { RMED } \\
(\mathrm{cm})\end{array}$ & $\begin{array}{c}\text { INT Rh } \\
(\mathrm{cm})\end{array}$ \\
\hline 877 & 0.3 & 0.2 & 1.4 & 1.2 & 0.8 & 5.7 \\
900 & 0.5 & 0.3 & 1.9 & 1.6 & 1.0 & 7.8 \\
929 & 0.4 & 0.3 & 1.7 & 1.7 & 1.0 & 7.6 \\
945 & 0.3 & 0.2 & 1.1 & 0.9 & 0.6 & 4.7 \\
\hline
\end{tabular}

ponents were removed from the adjusted coordinates of the GPS week 945 (1998) with respect to the GPS week 929 ones (1997B).

The observed coordinate differences between pairs of repeated surveys were submitted to the significance analysis by DENETGPS (Crespi, 1996). This software performs a testing procedure based on the Fisher variate (Koch, 1987) that separates the sites into two groups, those whose coordinates are significantly changed with respect to the error ellipsoids from those whose coordinates are not changed. DENETGPS was successfully tested in a suitably designed experiment by producing known displacements on one site of a GPS network (Crespi and Riguzzi, 1998).

The deformation analysis was performed on the consecutive adjusted coordinate sets corresponding to the time spans 1997A-1996, 1997B-1997A and 1998-1997B reported in table I.

Table IV shows the results of the analysis at $1 \%$ significance level in which the experimental Fisher values $F_{e}$ are compared with the threshold value $F_{t}$; sites with $F_{e}>F_{t}$ exhibit significant coordinate differences.

Note that the large value shown in the last comparison by the site of UPAD appears quite suspicious (last column, $F_{e}=114.84$ ). In terms of displacement correspond to a $2 \mathrm{D}$ vector magnitude of $(1.0 \pm 0.1) \mathrm{cm}$ and azimuth of $109^{\circ}$ (table V). Unfortunately, this jump appears in the same time interval as the UPAD antenna change dated December 23, 1997. The suspicious step spoils the whole analysis of the last comparison, wrongly conditioning the deformation analysis. To avoid any misinterpretation, UPAD was not considered in the last time span, thus achieving noticeably different $F$ values (last two columns). This fact shows, in spite of the care used in permanent GPS installation, how the results may be strongly conditioned by site (often antenna/receiver) problems (Springer et al., 1997).

Taking into account that the sites TORI and UPAD were not considered in the first and last comparisons respectively, the results of the deformation analysis may be globally sum- 
Table IV. Deformation analysis (1\% significance level).

\begin{tabular}{ccccc}
\hline Site & $\begin{array}{c}1997 \mathrm{~A}-1996 \\
F_{t}=3.89\end{array}$ & $\begin{array}{c}1997 \mathrm{~B}-1997 \mathrm{~A} \\
F_{t}=4.72\end{array}$ & $\begin{array}{c}1998-1997 \mathrm{~B} \\
\text { without UPAD } \\
F_{t}=3.89\end{array}$ & $\begin{array}{c}1998-1997 \mathrm{~B} \\
\text { with UPAD } \\
F_{t}=4.72\end{array}$ \\
\hline MEDI & $\mathbf{6 3 . 1 2}$ & $\mathbf{3 8 . 4 7}$ & $\mathbf{1 3 . 8 4}$ & $\mathbf{2 0 . 5 3}$ \\
CAGL & 3.69 & 0.35 & 1.75 & $\mathbf{7 . 8 2}$ \\
NOTO & $\mathbf{5 . 3 8}$ & $\mathbf{8 . 3 3}$ & 1.78 & $\mathbf{3 4 . 6 2}$ \\
WTZR & 3.18 & 2.10 & 0.04 & 1.43 \\
GRAZ & $\mathbf{4 . 6 7}$ & 0.17 & 0.97 & 0.04 \\
ZIMM & 1.40 & 0.74 & 0.40 & 0.00 \\
UPAD & 0.08 & 2.07 & - & $\mathbf{1 1 4 . 8 4}$ \\
TORI & - & 1.80 & 0.07 & 1.59 \\
MATE & $\mathbf{1 0 . 8 3}$ & $\mathbf{6 . 9 0}$ & 2.31 & $\mathbf{2 3 . 1 8}$ \\
\hline
\end{tabular}

Table V. 2D vector displacements, azimuth and rms.

\begin{tabular}{|c|c|c|c|c|c|c|c|c|}
\hline \multirow{2}{*}{ Site } & \multicolumn{2}{|c|}{ 1997A-1996 } & \multicolumn{2}{|c|}{ 1997B-1997A } & \multicolumn{2}{|c|}{$\begin{array}{c}\text { 1998-1997B } \\
\text { without UPAD }\end{array}$} & \multicolumn{2}{|c|}{$\begin{array}{l}\text { 1998-1997B } \\
\text { with UPAD }\end{array}$} \\
\hline & $\mathrm{cm}$ & deg & $\mathrm{cm}$ & $\operatorname{deg}$ & $\mathrm{cm}$ & deg & $\mathrm{cm}$ & deg \\
\hline MEDI & $0.8 \pm 0.1$ & 135 & $0.8 \pm 0.1$ & 9 & $0.3 \pm 0.1$ & 125 & $0.5 \pm 0.1$ & 124 \\
\hline CAGL & $0.1 \pm 0.2$ & 141 & $0.2 \pm 0.2$ & 39 & $0.2 \pm 0.2$ & -146 & $0.5 \pm 0.2$ & 153 \\
\hline NOTO & $0.3 \pm 0.2$ & 7 & $0.4 \pm 0.2$ & -38 & $0.1 \pm 0.2$ & 13 & $0.5 \pm 0.2$ & 87 \\
\hline WTZR & Fixed & - & Fixed & - & Fixed & - & Fixed & - \\
\hline GRAZ & $0.3 \pm 0.1$ & -38 & $0.1 \pm 0.1$ & 67 & $0.1 \pm 0.1$ & 176 & $0.1 \pm 0.1$ & 136 \\
\hline ZIMM & $0.2 \pm 0.1$ & -125 & $0.2 \pm 0.1$ & 104 & $0.0 \pm 0.1$ & 11 & $0.1 \pm 0.1$ & 164 \\
\hline UPAD & $0.1 \pm 0.1$ & -83 & $0.2 \pm 0.1$ & 49 & - & - & $1.0 \pm 0.1$ & 109 \\
\hline TORI & - & - & $0.2 \pm 0.1$ & 121 & $0.0 \pm 0.1$ & -172 & $0.2 \pm 0.1$ & 162 \\
\hline MATE & $0.3 \pm 0.2$ & 16 & $0.5 \pm 0.2$ & -8 & $0.4 \pm 0.2$ & 82 & $0.5 \pm 0.2$ & 97 \\
\hline
\end{tabular}

marized as follows:

- CAGL, GRAZ, TORI, UPAD, ZIMM and WTZR do not show any significant coordinate differences.

- MEDI exhibits significant coordinate differences in all the comparisons with the largest $F_{e}$ values of the whole network.

- NOTO and MATE show significant coordinate differences in the first two comparisons, and in the last comparison, although not significant, they have the largest $F_{e}$ values after MEDI (it is necessary to remember here that the time span pertaining to the last comparison is about $2 / 3$ of the other two).

\section{Displacements and velocities}

Taking into account the results of the deformation analysis, the consecutive $2 \mathrm{D}$ vector displacements, their azimuths and the corresponding error ellipses on the local tangent plane at $99 \%$ confidence level were computed with respect to WTZR (table V) and reported in fig. 1.

Although the more general representations of vector displacements are to compute them with respect to the datum baricentrum, i.e. the baricentrum of the stable sites (Brunner et al., 1981; Koch, 1984), the choice to compute vectors with respect to one site is correct (Teunis- 


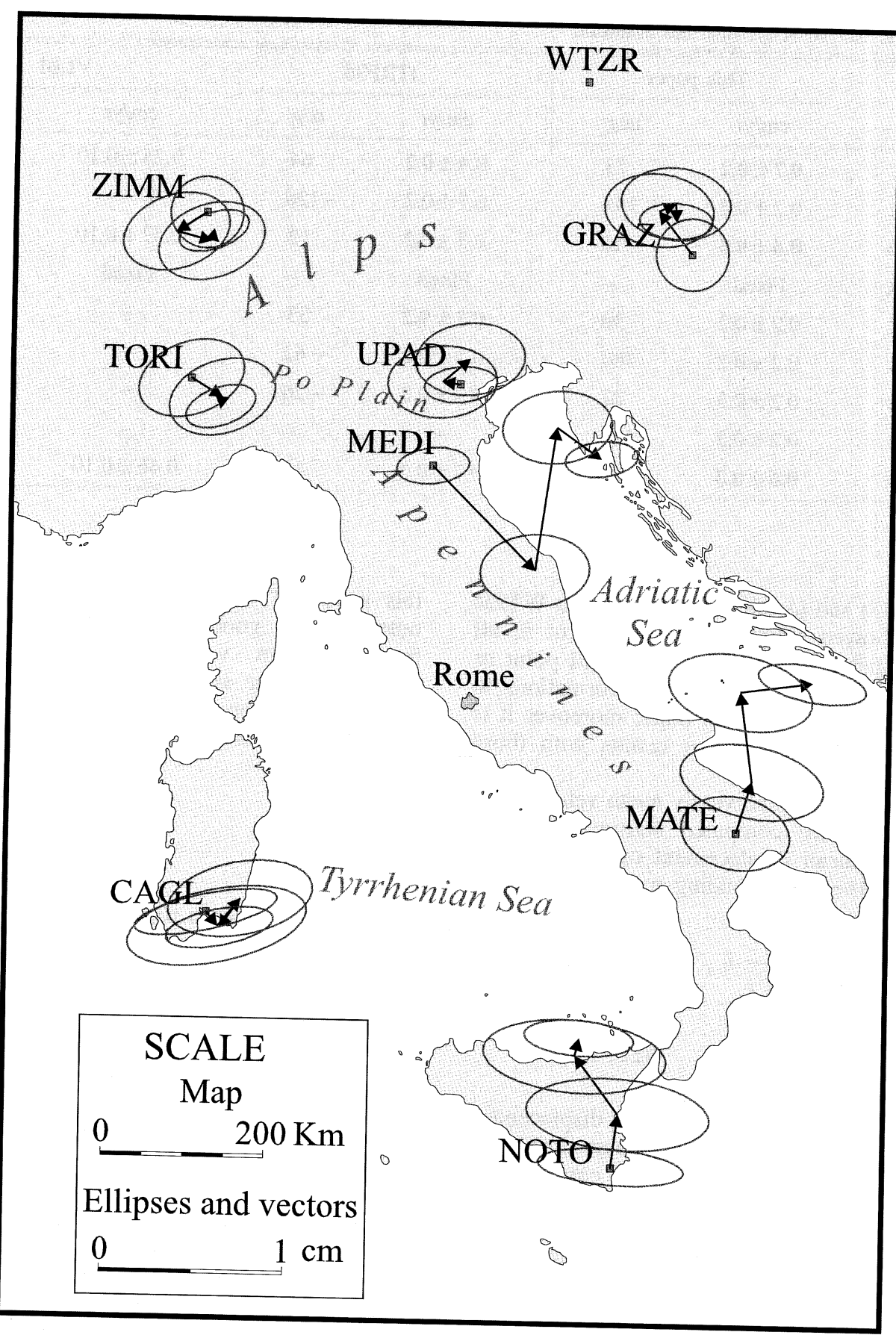

Fig. 1. 2D vector displacements and error ellipses at $99 \%$ confidence level computed with respect to WTZR
for the three time spans. 
Table VI. 2D velocity, rms and azimuth.

\begin{tabular}{ccccccccc}
\hline \multirow{2}{*}{ Site } & \multicolumn{2}{c}{ This paper } & & \multicolumn{2}{c}{ ITRF96 } & & \multicolumn{2}{c}{ VLBI } \\
\cline { 2 - 3 } \cline { 7 - 8 } \cline { 7 - 8 } & $\mathrm{cm} / \mathrm{yr}$ & $\mathrm{deg}$ & & $\mathrm{cm} / \mathrm{yr}$ & $\mathrm{deg}$ & & $\mathrm{cm} / \mathrm{yr}$ & $\mathrm{deg}$ \\
\hline MEDI & $\mathbf{0 . 7} \pm \mathbf{0 . 2}$ & 73 & & $0.4 \pm 0.2$ & 64 & & $0.25 \pm 0.10$ & 57 \\
CAGL & $0.2 \pm 0.3$ & 135 & & $0.7 \pm 0.2$ & -126 & & - & - \\
NOTO & $\mathbf{0 . 6} \pm \mathbf{0 . 3}$ & -16 & & $0.7 \pm 0.2$ & 12 & & $0.57 \pm 0.10$ & 7 \\
WTZR & Fixed & - & & Fixed & - & & Fixed & - \\
GRAZ & $0.2 \pm 0.2$ & 30 & & $0.2 \pm 0.2$ & 33 & & - & - \\
ZIMM & $0.2 \pm 0.2$ & 180 & & $0.1 \pm 0.2$ & -62 & & - & - \\
UPAD & $0.2 \pm 0.3$ & 27 & & $0.3 \pm 0.2$ & -26 & & - & - \\
TORI & $0.2 \pm 0.3$ & 116 & & - & - & & - & - \\
MATE & $\mathbf{0 . 6} \pm \mathbf{0 . 3}$ & 10 & & $0.7 \pm 0.2$ & 21 & & $0.46 \pm 0.10$ & 29 \\
\hline
\end{tabular}

sen, 1985) and justified by the fact that WTZR never showed significant displacement in all the comparisons; from a geophysical point of view the meaning is to refer deformations to the stable inner Eurasian plate; moreover it is necessary to compare our results with those from other papers.

With this aim, the yearly mean velocities of each site were computed starting from the weighted mean displacement of each component and the corresponding rms defined by

$$
d_{i}=\frac{\sum_{k=1,3} \frac{1}{\sigma_{i, k}^{2}} d_{i, k}}{\sum_{k=1,3} \frac{1}{\sigma_{i, k}^{2}}} \pm \frac{1}{\sqrt{\sum_{k=1,3} \frac{1}{\sigma_{i, k}^{2}}}}
$$

where $i=\varphi, \lambda$ and $d_{i, k}$ is the $k$ th displacement of the $i$ th component over the same time interval. The final mean velocity was computed by propagating the resulting vector over one year. Table VI shows the mean velocities $(\mathrm{cm} / \mathrm{yr})$, the corresponding rms and their azimuths from this paper, from ITRF96 solutions (Boucher, 1998) and from VLBI solutions (Tomasi, 1997), computed with respect to WTZR.

Figure 2 shows the site mean velocities $(\mathrm{cm} / \mathrm{yr})$ computed with respect to WTZR from this paper, ITRF96 and VLBI. It has to be noted that a good agreement among these three different velocity determinations is achieved for the sites that showed significant displacements; on the contrary, sites without significant displacements may display diverging results.

\section{Discussion}

This study made a rigorous analysis of GPS data samples of nine permanent tracking sites.

The network is composed of six sites located in Italy (CAGL, MATE, MEDI, NOTO, TORI and UPAD) and another three located outside Italy, on the inner Eurasian plate (GRAZ, ZIMM and WTZR).

The deformation analysis shows significant displacements at MATE, MEDI and NOTO. No globally significant displacements are exhibited by CAGL, GRAZ, TORI, UPAD, ZIMM and WTZR within the analyzed time span.

The 2D displacement vectors show a noisy trend for all the significant sites with a higher level at MEDI (table V, fig. 1). The noise may be related both to local environmental conditions, as hypothesized by Springer et al. (1997) for MEDI, and to unmodelled residual correla- 


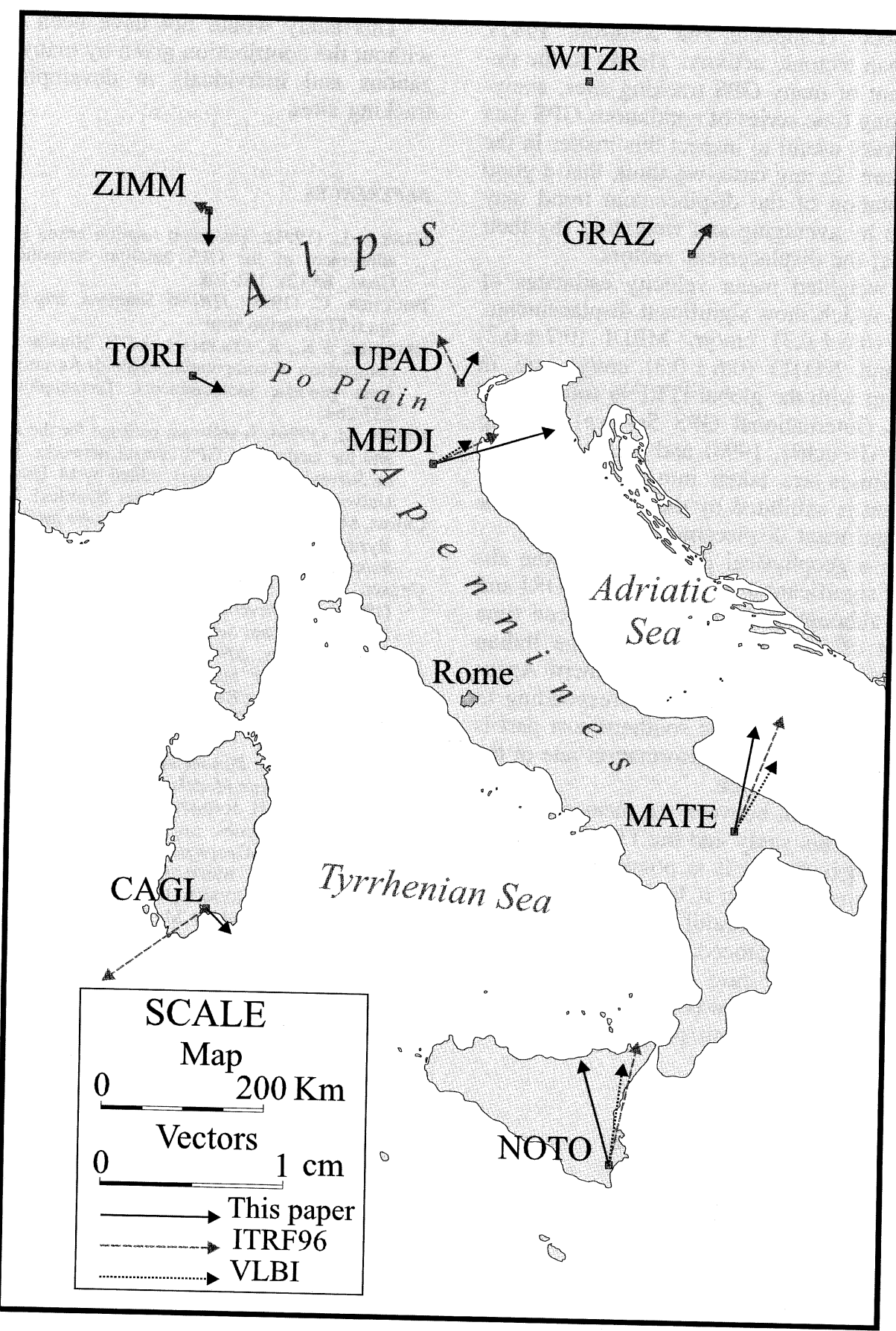

Fig. 2. Comparison of the site mean velocities $(\mathrm{cm} / \mathrm{yr})$ computed with respect to WTZR from this paper,
ITRF96 and VLBI. 
tion effects (Langbein and Johnson, 1997), rather than tectonic activity. Thanks to the development of many GPS tracking sites, analyses on long time series of continuous GPS data will be very useful to inspect this matter in the near future. In any case, we think that a good representation of the displacement trend may be given by averaging and weighting (by their own rms) the displacement vectors.

The weighted mean velocity estimates of the sites which show significant displacements, MATE $(0.6 \pm 0.3) \quad \mathrm{cm} / \mathrm{yr}$, MEDI $(0.7 \pm 0.2)$ $\mathrm{cm} / \mathrm{yr}$ and NOTO $(0.6 \pm 0.3) \mathrm{cm} / \mathrm{yr}$, are in agreement with the global ITRF96 solutions of the IGS (International GPS Service) tracking network (Boucher, 1998) and VLBI, provided error ellipses are taken into account. Minor agreement is exhibited by the sites that do not show significant displacements (fig. 2).

From a geophysical point of view, the absence of significant displacements at TORI and UPAD, although estimated on a reduced time span, let us infer that, at present, the Italian peninsula is separated by the Northern Apennines area in a stable region corresponding to the Po plain area and the southernmost part is subjected to the highest deformation rate of the whole investigated area.

The agreement between the mean velocities obtained in this study and the ITRF96 investigations suggests that it is possible to achieve significant results even if limited data sets are analyzed, provided careful data check and rigorous processing procedures are carried out. Emblematic is the case of UPAD whose antenna changing effect was discovered $a$ posteriori using such rigorous procedure.

\section{Acknowledgements}

The authors are grateful to P. Baldi for the helpful suggestions given in reviewing the manuscript.

The authors wish to acknowledge C. Noll product manager of the CDDIS and P. Colucci of the Italian Space Agency (ASI) for providing GPS observations.
This study would not have been possible without the contribution given by many organizations and individuals in developing GPS tracking sites.

\section{REFERENCES}

BÁNYAI, L. (1991): Treatment rotation errors in the final adjustment of the GPS baseline components, Bull. Geod., 65 (2), 102-108.

BouCHER, C. (1998): ITRF96 Solutions, http://lareg.ensg. ign.fr/ITRF/itrf96.html.

BrunNeR, F.K., R. Coleman and B. Hirsch (1981): A comparison of computation methods for crustal strains from geodetic measurements, Tectonophysics, 218, 237-264.

CREsPI, M. (1996): A software package for the adjustment and the analysis of GPS control networks, in Reports on Surveying and Geodesy, edited by M. UNGUENDOLI, University of Bologna (Edizioni Nautilus), 237-264.

CRESPI, M. and F. RIGUZZI (1998): Software available for analyzing GPS deformation, Eos n., p. and Eos electronic Supplement, http://www.agu.org/eos_elec/98059e.html.

Crespi, M., B. Marana, F. SAnsò and D. Sguerso (1993): GPS leveling and the Geoid, in Proceedings 2nd International Seminar on GPS in Central Europe, Penc, Hungary, 209-228.

KocH, K.R. (1984): Statistical tests for detecting crustal movements using Bayesian inference, NOAA Technical Report NOS NGS 29, National Geodetic Survey, Rockville, MD.

KocH, K.R. (1987): Parameter Estimation and Hypothesis Testing in Linear Models (Springer-Verlag, Berlin).

LANGBEIN, J. and H. JoHNSON (1997): Correlated errors in geodetic time series: implications for time dependent deformation, J. Geophys. Res., 102, 591-603.

Rothacher, M., G. Beutler, W. Gurtner, E. BrockMANN and L. MERVART (1995): Bernese GPS Software Version 4.0, edited by M. ROTHACHER and L. MERVART, University of Berne, Switzerland.

SPringer, T.A., W. GURTNER, M. Rothacher and S. SCHAER (1997): EUREF Activities at the CODE Analysis Center. Paper presented at International Seminar on GPS in Central Europe, Penc, Hungary, May 7-9 (in press).

TEUNISSEN, P.J. (1985): Zero order design: generalized inverses, adjustment, the datum problem and $S$-transformations, in Optimization and Design of Geodetic Networks, edited by E.W. GRAFAREND and F. SANSÒ (Springer-Verlag, Berlin), 11-55.

TomAsI, P. (1997): Geodetic VLBI in Europe: the status of the network and the recent results, in Proceedings of the Meeting "Geodesia Spaziale nel Mediterraneo», Stazione Astronomica di Cagliari, Cagliari 27-28 febbraio 1997, 5-22.

(received April 14, 1998; accepted May 30, 1998) 